\title{
Squeezing some More from the Eurth
}

Ir might have been thought that once seismology had presented a clear picture of the elastic properties of the Earth it could disappear gracefully from the scone. The trouble, or to the insider the virtue, is that the more carefully the Earth is studied the less it is possible to fit its elastic properties into any simple spherically symmctric model. The structural difference between the upper mantle under continents and under oceans is bclieved to extend to a depth of at least $600 \mathrm{~km}$. Substantial slices of the mantle in which deep earthquakes occur (for example, round the Pacific) are known to be out of thermal equilibrium with their surroundings. The techniques for determining earthquake mechanisms have been improved beyond all recognition in the past three or four years. And commanding increasing support is the earthquake prediction programme, which is quietly being renamed the earthquake control programme. So the future still holds lots of prizes for the seismologist.

New problems are very often best attacked with new tools and in the past two years there has been a spato of instrumental developments. Coverage of the world with short period ( $1 \mathrm{~s}$ ) instruments is now reasonably good. These instruments are of particular use in location of events, and work with magnifications up to a million. There are several "holes" in global coverage - the Pacific Ocean, the Arctie, Africa-but stations on Pacific Islands, in Northern Canada and Siberia and in Kenya, Ethiopia, Egypt and South Africa help to plug the gaps. The longer period instruments (10 s up to thousands of seconds) are less thick on the ground and arc particularly inadequate for periods greater than $40 \mathrm{~s}$. Such seismometcrs are of use in studies of carthquake mechanism, distribution of strain patterns before and after earthquakes and study of the deeper structure of the Earth by long-period surface waves and free vibrations of the Earth. It gocs without saying that they are of considerable value in nuclcar test detection. The number of such scismometers in use, however, is now increasing rapidly. Particularly notoworthy are the novel designs that are being used.

At loast four new types of laser seismometer have becn designed in the past two or three years. These are strain measuring devices which work as interferometers with automatic fringe detection. Strains as small as 1 in $10^{\prime \prime}$ may be measured and laser seismometers with a broadband response are extremely versatile seismometers. Because the optical paths are usually in vacuo, environmental control need not be stringent. Although two or three work in disused railway tunnels, one $(800 \mathrm{~m}$ long) sits on the ground basking in the Californian sunshine.

Last year some impressive new results were demonstrated by Molnar et al. (Nature, 224, 1268; 1969) using careful environmental control of a conventional long period seismometer and improved amplification devices. Undoubtedly these new Lamont seismometers will open up fields of research in a part of the spectrum so far very inadequately treated.

In this issue (page 343 ), Block et al. describe some of the results recently obtained with the new accelerometer developed at Scripps during the past three or four years. This instrument is interesting in that it was designed by physicists to see how good an accelero. meter could be made, rather than by geophysicists with particular problems in mind. The result is quite remarkable. Block et al. report very clear oxcitation of the free oscillations of the Earth from a magnitude 6.5 earthquake. There are generally at least twenty earthquakes of this magnitude or greater every ycar. To put this achievement in perspective we may note that free oscillation observations had only previously been possible from earthquakes with magnitudes greater than $7 \cdot 7$, and that only two earthquakes (Chile, 1960; Alaska, 1964) have received widespread attention. It is reported that, since the New Hebrides earthquake, several other earthquakes have allowed the measurement of free oscillations.

It seems that a new era of instrumental develop. ment and increased seismological knowledge is opening. The subject is clearly going to be as lively in the next docade as it was in the last.

\section{COMETS \\ Another Hydrogen Cloud}

\section{by our Astronomy Correspondent}

A SECOND example of a cloud of hydrogen surrounding the head of a comet was reported last week by NASA. Observations from one of the Orbiting Geophysical Observatories show that comet Bennett which first appeared over Australia in January is immersed in a cloud of hydrogen about eight million miles acrossten times the diameter of the Sun.

This follows the news of a hydrogen cloud surrounding the comet Tago-Sato-Kosaka, announced by NASA earlier this year from data obtained with the Orbiting Astronomical Observatory, OAO 2 (see Nature, 225, $413 ; 1970)$. The size of the cloud around Tago-SatoKosaka was said to be comparable with the size of the Sun.

Although the hydrogen clouds are unexpected, they are fairly simple to account for on the current model for cometary nuclei. As the comet approaches the Sun some of the gases which are contained in the mass of mineral particles and frozen gases which make up the nucleus would be expected to boil off. The problem is that the coma of gas surrounding the nucleus, which is recognized to be the result of this process, is tiny by comparison with the hydrogen clouds now observed.

The reason why the hydrogen clouds have not been observed before is that the ultraviolet radiation which 